\title{
The beginnings of tuberculosis pharmacological treatment
}

\author{
Raúl Cicero-Sabido ${ }^{1}$ and Guillermo Meléndez-Mier ${ }^{2}$
}

${ }^{1}$ Universidad Nacional Autónoma de México, Faculty of Medicine; ${ }^{2}$ Secretaría de Salud, Hospital General de México, Directorate of Research, Ciudad de México, Mexico

The solution of a complex problem, such as tuberculosis, should be seen as an incentive to develop new ways to work together to the benefit of the community and man himself. The Lord hath created medicines out of the Earth; and he that is wise will not abhor them.

[ECCLESIASTICUS, XXXVIII, 4]

\begin{abstract}
The appearance of new anti-tuberculosis drugs such as bedaquiline and delamanid makes it impossible not to remember that the first strictly controlled medical trials of tuberculosis treatment were published in two rigorously researched outstanding articles that can be qualified as historical. In 1948, streptomycin was formally studied as an efficacious anti-tuberculosis drug. In 1952, another trial compared streptomycin-paramino salicylic acid with isoniazid, by means of which the first bases of pharmacological tuberculosis treatment were established.
\end{abstract}

KEY WORDS: Streptomycin. Isoniazid. Para-aminosalicylic acid. Drug-resistance.

With the appearance of new anti-tuberculosis drugs such as bedaquiline and delaminid, ${ }^{1}$ it is important to remember that the first strictly controlled assays of tuberculosis medical treatment were published in two excellent and rigorous scientific research articles that can be qualified as historical. The discovery of streptomycin by Selman Waksman in 1944 and its use as the first efficacious anti-tuberculosis drug was formally studied in $1948^{2}$ and was followed in 1952 by another trial that compared streptomycin-para-aminosalicylic acid with isoniazid, ${ }^{3}$ whereby the first bases of tuberculosis pharmacological treatment (chemotherapy) were established. At the same time, the appearance of drug resistance developed by Mycobacterium tuberculosis was observed, which constitutes a therapeutic challenge.

In the first trial of 1948, 11 experts of seven important hospitals, coordinated by Geoffrey Marshall from the Brompton Hospital in London, participated and designed the protocol on the effects of streptomycin on pulmonary tuberculosis by comparing bed rest with administration of the drug: a significant number of patients died with the former measure. Of those who received streptomycin, many showed considerable clinical and radiological improvement at 3 months, although, in other cases, the bacillus was found to have developed resistance to the antibiotic at different moments of treatment. This research was endorsed by the British Medical Research Council as an example of a trial with results assessed with random selection statistical methods. ${ }^{4}$

In 1952, "TREATMENT of pulmonary tuberculosis with isoniazid; an interim report to the Medical Research Council by their Tuberculosis Chemotherapy Trials Committee" was published, where the results of the research coordinated by Marc Daniels and Wallace Fox comparing isoniazid and streptomycin-para-aminosalicylic acid were reported. In this trial, it was concluded that isoniazid alone is not more effective than streptomycin-para-aminosalicylic acid, and $71 \%$ of Mycobacterium tuberculosis strains were found to have become resistant after 3 months of treatment.
Correspondence:

Raúl Cicero-Sabido

E-mail: rc1neumo@unam.mx
Date of reception: 31-07-2017

Date of acceptance: 10-08-2017

DOI://dx.doi.org/10.24875/GMM.M18000199
Gac Med Mex. 2018;154:527-528

Contents available at PubMed www.gacetamedicademexico.com 
The performance of both these protocols was highly significant in the fight against tuberculosis; they indicated that the combination of several drugs might achieve the cure. The combination of isoniazid, rifampicin, pyrazinamide and ethambutol is the basis of anti-tuberculosis strictly supervised treatment that is currently employed all over the world. Resistance, multi-drug resistance and extreme resistance persist as a real challenge to achieve tuberculosis cure.

Each new anti-tuberculous drug must be subjected to a strict protocol, even in experimental animals, to demonstrate its efficiency and to determine Mycobacterium tuberculosis susceptibility and whether it shows resistance that can neutralize the medication therapeutic effect. Approval by the World Health Organization and the Food and Drug Administration is vital for clinical application and marketing of any drug. ${ }^{5}$

In tuberculosis, not only discovery and use of new drugs are essential, but working for a world where all its inhabitants have access to health services, with anti-tuberculosis fight programs strictly carried out with effective drugs, and where poverty, ${ }^{6}$ which is linked to tuberculosis and makes it difficult to detect and treat the disease, is alleviated. ${ }^{7}$ Given the serious inequality that prevails in several countries, ${ }^{7}$ eradication of tuberculosis is perhaps a utopia. ${ }^{7}$ The search for effective new drugs should continue as a hope for patients infected with strains of resistant mycobacteria. The example of those who have done it is present.

\section{References}

1. Bayfield A, editor. Martindale. The complete drug reference. Pharmaceutical Press: Londres; 2014.

2. Streptomycin treatment of pulmonary tuberculosis. A medicaßresearch council investigation. Br Med J. 1948;2(4582):769-782.

3. TREATMENT of pulmonary tuberculosis with isoniazid; an interim report to the Medical Research Council by their Tuberculosis Chemotherapy Trials Committee. Br Med J. 1952;2(4787):735-746.

4. Chalmers I. Why the 1948 MRC trial of streptomycin used treatment allocation based on random numbers. J R Soc Med. 2011;104(9):383-386.

5. Michinson D, Davies G. The chemotherapy of tuberculosis: past, present and future. Int J Tuberc Lung Dis. 2012;16(6):724-732.

6. World Health Organization. Global tuberculosis report 2015. Suiza: World Health Organization; 2016.

7. Ross A, Rosenberg P, Dye C, Prasad A. A world cities and the end of TB. Trans R Soc Trop Med Hyg. 2016;110(3):151-152. 